\title{
Effect of water medium upon the process of ore disintegration in wet self-grinding mills
}

\author{
Vitalii Monastyrskyi ${ }^{1}$, Serhii Monastyrskyi ${ }^{1}$, and Borys Mostovyi ${ }^{1, *}$ \\ ${ }^{1}$ Institute of Geotechnical Mechanics named by N. Poljakov of National Academy of Sciences of \\ Ukraine, 49005, Dnipro, Simferopolska Str., 2a, Ukraine
}

\begin{abstract}
This article presents the results of theoretical and experimental studies on the processes of destruction ore in wet self-grinding mills under the influence of the aquatic environment on it. The equivalent circuit for determining the stress-strain state of a large piece (ball) exposed to the load at an angle of repose of the material in motion are justified. A uniformly distributed pressure acts on the body of arbitrary shape, depending on the hydrostatic pressure of the pulp inside the drum and the force of hydrodynamic resistance). It is assumed that a large piece has the shape of a sphere with a uniform structure, but in some of its parts there are cavities filled with an incompressible fluid. Under the action of these forces inside the cavity wedging effects of water occur, the effect of which on the strength ores depends on the expansion force and the angle of the micro crack. The experimental researches are done under laboratory and industrial conditions. It was established that the disintegration of kimberlitic ore in MMC is significantly influenced by the characteristics of the aquatic environment $\mathrm{pH}$ and $E h$, the values of which vary from 3.2 to 7.0 and from -500 to $+1020 \mathrm{mV}$.
\end{abstract}

\section{Introduction}

Dressing plants, which process diamond-bearing raw material, use wet self-grinding mills (WSGM) to crush and grind bulk commodity with the coarseness of 300-900 mm; the plants help liberate diamonds, remove films on their surface, and grind initial ore up to the required processing depth. Currently, ore-mining enterprises often use WSGM of the diameter from 5 up to $10.5 \mathrm{~m}$ produced by Syzran plant and such foreign companies as Svedala and Rockcyle. According to the studies by Yakutniproalmaz, the mills operate at $n_{o}=0.75 n_{c r}$ rotation frequency ( $n_{c r}$ is the critical frequency of drum rotation), coefficient of drum charge being from 0.3 to $0.45, \mathrm{H}: \mathrm{L}>1: 0.8$ ratio, and temperature of process water being $4^{\circ}$ or equal to the environmental one at the ore surface taking into consideration permafrost conditions.

In the process of drum rotation, water medium is heated and the obtained heat energy is used for the material thawing along with its dissipation in the environment. In terms of long-term thawing, diamond-bearing ore loses its iceness, strength, and durability which results in the fact that the lumps are broken even in terms of insignificant dynamic loading.

\footnotetext{
*Corresponding author: Sly3@i.ua
} 
However, papers $[1,2]$ determine that in the context of near-lifter and waterfall operating modes of WSGM, lumps are raised by height $H$ during the drum rotation and interact with the load core (totality of lumps) or components of the drum liner at $\beta$ angle under the gravity force. In this case, dynamic loads remain to be significant and water medium has not time to effect considerably the strength and durability of lumps.

Paper [3] represents computational scheme and analytical expressions to determine energy losses of a falling lump at the moment of its interaction with the water plane depending upon the lump mass, its falling height, and interaction angle. It has been determined that water layer reduces the velocity of lump falling by $25-30 \%$; that results in the increased dynamic loads on separate lumps in the center of WSGM and improves diamond integrity.

\section{Statement of the research problem}

Depending on $E h, p H$ values, water medium is capable of penetrating into the lumps through cracks formed in the mother rock (germinal ones) or due to the effect of a blast wave. Argillaceous and ochreous minerals being practically always available in mineralogical composition of the deposits of diamond-bearing raw material, form specific films on the mineral surfaces which contact with them at the molecular level. According to [4], such films are capable of widening (wedging) the microcracks though which water penetrates into a lump filling the available cavities. When the lump and obstacles interact, films are destructed, microcracks are closed at the expense of material elasticity, and a lump may be represented as a sphere inside of which there are minor cavities filled with the incompressible liquid. Effect of the water medium characteristics upon the process of rock and ore breaking inside the WSGM drum was studied both theoretically and experimentally.

\section{Solution of the problem and its analysis}

A problem of determining stress and strain state of a lump in the process of its effecting by water medium and dynamic loads was solved theoretically while a problem of selecting basic characteristics of water medium in terms of which ore and rock breaking would be optimal was solved experimentally.

Figure 1, a,b demonstrates computational schemes to determine stress and strain state of a lump (sphere) located on a lifter plane and being effected by the loads at the angle of natural slope of the material in motion. In this case, an arbitrary shaped body is effected by equally distributed pressure $P_{k}$ which value is defined depending upon hydrostatic pressure of the pulp $P_{p}$ inside a drum and force of hydrodynamic resistance $P_{r}$ in terms of lump movement along with the lifter at velocity

$$
V_{l}=\frac{\pi n_{d} D_{d}}{60}
$$

where $n_{d}$ is frequency of drum rotation; $D_{d}$ is drum diameter.

To formalize the computational scheme, following factors were assumed:

- lump shape is taken as a sphere which projection to the plane perpendicular to the force effect is determined from expression $S=\pi D^{2} / 4$, where $D$ is sphere diameter;

- we should study three-axial compression of a lump under the effect of uniform pressure $P_{k}$; 
- stress and strain state of the sphere is considered at the moment when forces of resistance and hydrostatic pressure have their maximum values (see Fig. 1);

- lifter elasticity is neglected, a lump is considered to be lying on a rigid base; and

- sphere is of homogeneous structure, however, its certain parts have cavities filled with incompressible liquid.

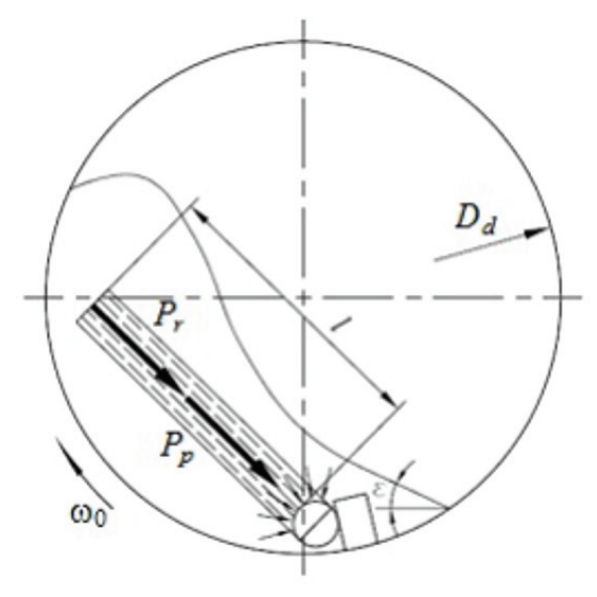

a

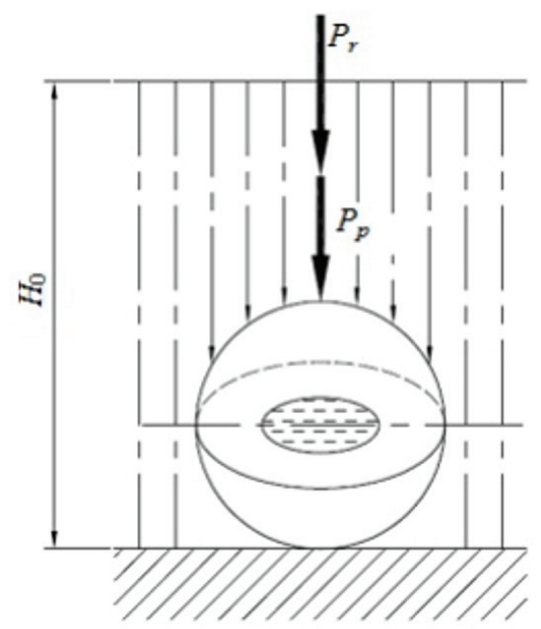

b

Fig. 1. Computational scheme to determine stress and strain state of a lump (sphere).

According to [5], pressure effecting the lump is determined from expression

$$
P_{k}=g \gamma_{p} H_{0}+K_{h d} \gamma_{h} V^{2} S,
$$

where $\gamma_{h}, \gamma_{p}$ are densities of hard and pulp masses respectively; $H_{0}$ is hydrostatic (maximum) pressure on the lump in the center of its mass; $g$ is free fall acceleration; $V$ is velocity of lump motion in the liquid; $K_{h d}$ is hydrodynamic coefficient of liquid resistance; and $S$ is area of the sphere projection on the interaction plane.

In terms of three-axial compression by $P_{k}$ pressure, any area inside the sphere experiences stresses equal to $P_{k}$ [6]; depending upon the cross-section area of a cavity filled with water, force $F=P_{k} F_{c}$ (where $F_{c}$ is cross-section area of cavity inside the sphere) will occur at any direction.

Figure 2 represents computational scheme to determine wedging effect of water inside the sphere. In this context, we consider that pressure from force $F$ is transmitted to a microcrack with $2 \alpha$ angle by means of $R$ forces which value is defined by projecting all the forces on axes $x$ and $y: R=F / 2 \sin \alpha$.

Effect of a wedging force upon the ore strength was determined experimentally under laboratory conditions using a plant for one-axial compression of samples from different ores and rocks; the plant was equipped with hydraulic press with $60 \mathrm{kN}$ and computer for simultaneous recording of compression force and deformation. The research methodology stipulated that tests were carried out involving following samples: dry, previously wetted, solid (Fig. 3,a), glued two-layer (Fig.3, b), and with a cavity (Fig. 3,c). Table 1 represents the results of tests for samples compression up to their breaking; Figures 4-6 demonstrate diagrams of sample compression and their photos after breaking. 


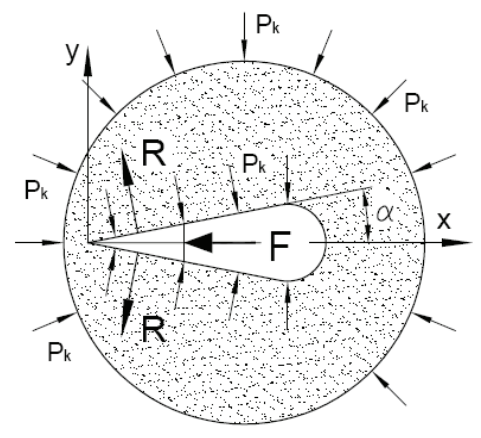

Fig. 2. Computational scheme to determine wedging effect of water.
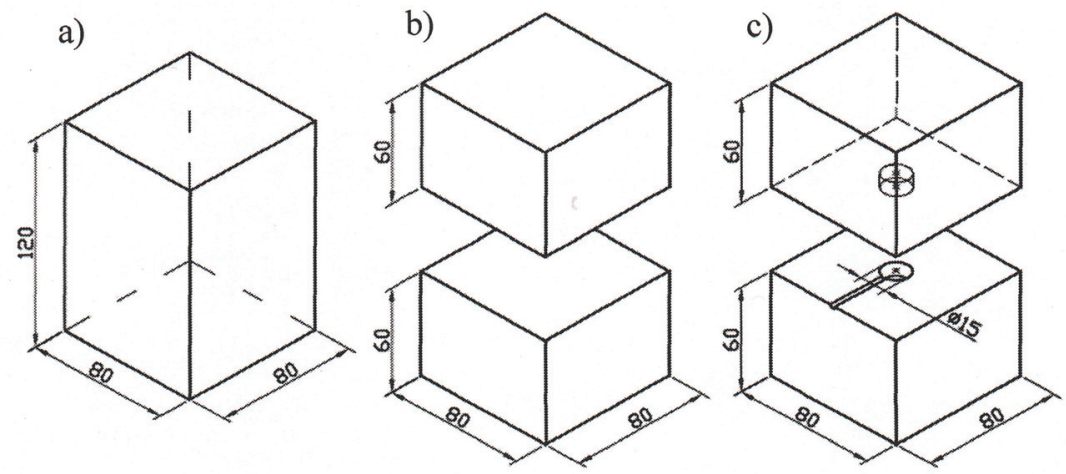

Fig. 3. Test samples: a) solid sample; b) cut sample; c) sample with a cavity.

Table 1. Results of sample testing for breaking compression.

\begin{tabular}{|c|c|c|c|c|c|c|}
\hline 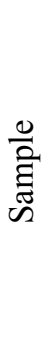 & Sample characteristics & 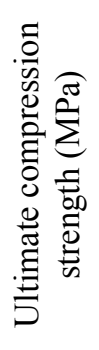 & 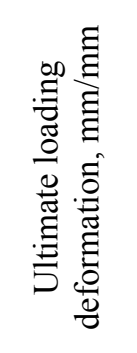 & 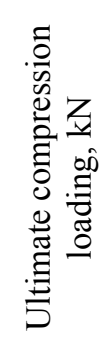 & 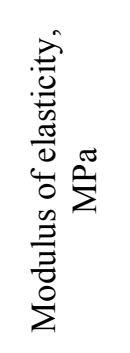 & 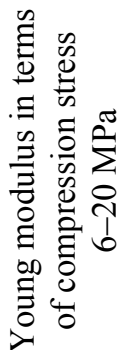 \\
\hline 1 & $80 \times 80 \times 120$, dry & 91.47 & 0.01437 & 630.01 & 6281.54 & 5850.81 \\
\hline 2 & $80 \times 80 \times 120$, dry & 91.46 & 0.01452 & 630.01 & 6209.54 & 5907.68 \\
\hline 3 & $80 \times 80 \times 120$ wet & 82.82 & 0.01439 & 570.43 & 6077.81 & 5093.89 \\
\hline 4 & $80 \times 80 \times 120$, wet & 57.76 & 0.00998 & 465.02 & 5163.72 & 5012.77 \\
\hline 5 & $80 \times 80 \times 120$, dry & 71.58 & 0.01363 & 493.01 & 5488.86 & 5106.93 \\
\hline 6 & $80 \times 80 \times 120$, wet & 51.25 & 0.00965 & 432.23 & 5098.72 & 5006.11 \\
\hline 7 & $80 \times 80 \times 120$, dry & 55.28 & 0.00974 & 443.11 & 5122.61 & 5010.18 \\
\hline 8 & $80 \times 80 \times 120$, wet & 47.38 & 0.00949 & 326.34 & 5083.19 & 4821.06 \\
\hline
\end{tabular}




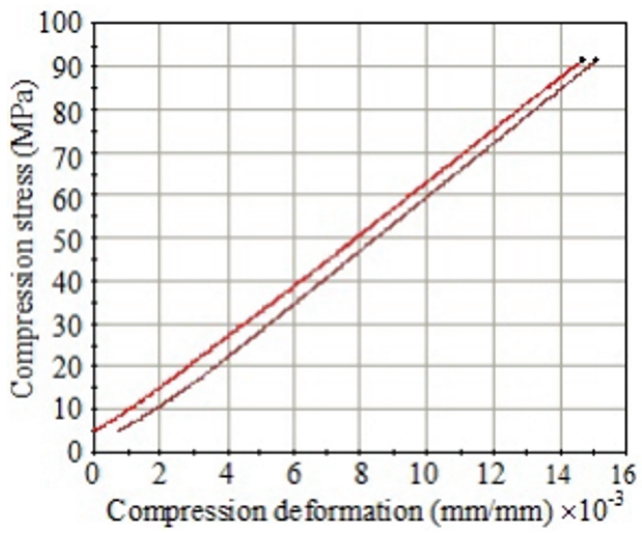

a

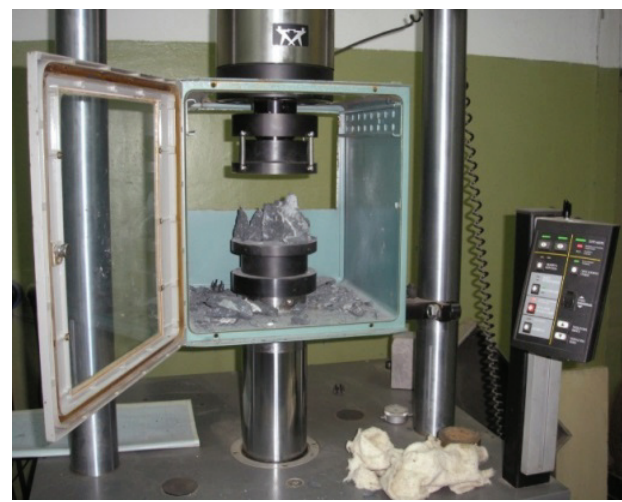

b

Fig. 4. Diagram of samples $\# 1$ and $\# 2$ compression (a) (see Table 1) and their photos in broken condition (b).

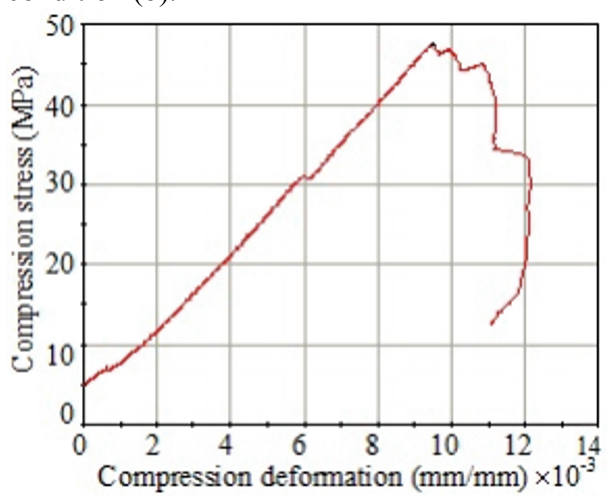

a

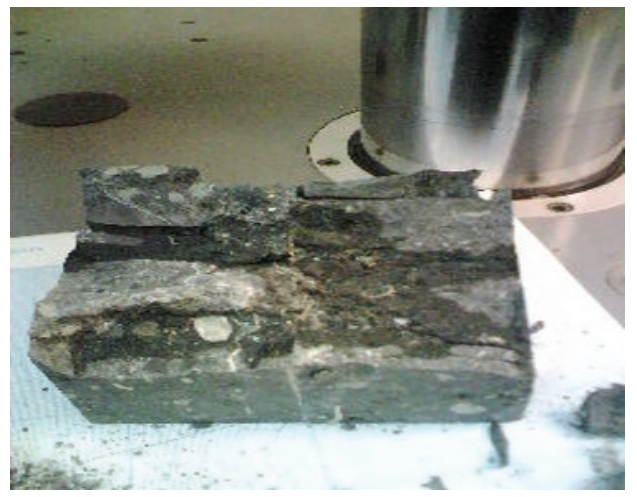

b

Fig. 5. Diagram of sample \#5 compression (a) (see Table 1) and its photo in broken condition (b).

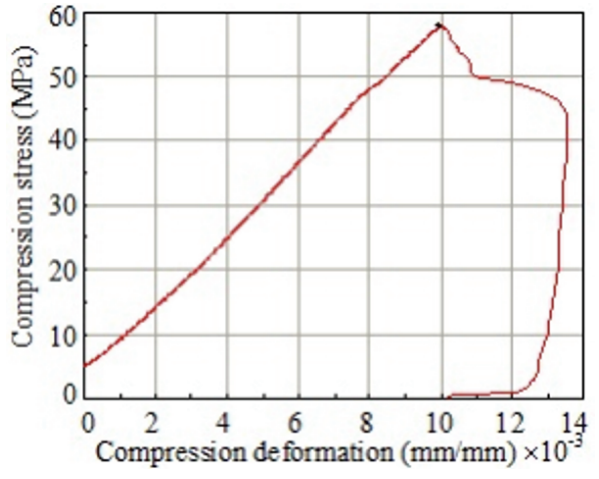

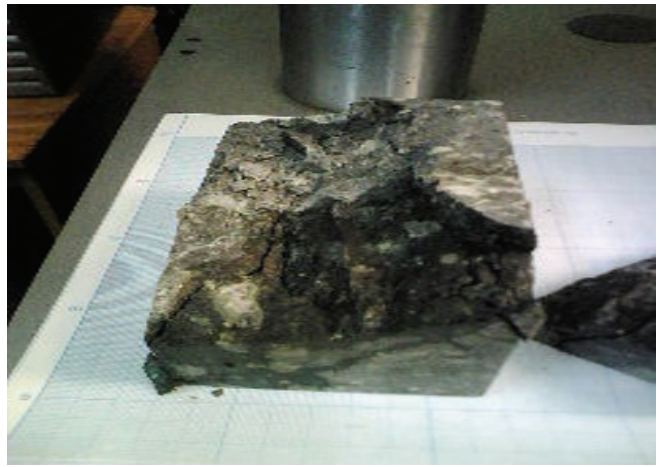

b

Fig. 6. Diagram of sample \#6 compression (a) (see Table 1) and its photo in broken condition (b).

Basing upon the performed studies, following conclusions may be drawn:

- while compressing dry and wet samples, stress is subject to a linear law depending upon their relative deformation: in terms of dry solid sample (see Fig. 4,a) - up to 0.015; in terms of wet solid sample (see Fig. 5,a) - up to 0.010; and in terms of wet sample with a cavity (see Fig. 6,a) - up to 0.009; 
- if sample condition is changed (dry, wet, with a cavity), ultimate breaking stresses are as follows (see Table 1): $91 \mathrm{MPa}$ - solid dry (samples \#1 and \#2); $83 \mathrm{MPa}$ - solid wet (sample \#3); $71 \mathrm{MPa}$ - dry two-layer (glued) without a cavity (sample \#5); $57 \mathrm{MPa}$ - wet two-layer (glued) without a cavity (sample \#4); $55 \mathrm{MPa}$ - dry with a cavity (sample \#7); and $48 \mathrm{MPa}$ - dry with a cavity (sample \#8).

Thus, water medium reduces strength of solid ore samples by $8-10 \%$; in case of wet samples with a cavity - the reduction is by $40 \%$ comparing to the solid ones.

Paper [7] determines experimentally the effect of water characteristics $E h$ and $p H$ upon the process of ore disintegration. The experiments were carried out in the apparatus of membraneless type in continuous-flow mode with process water parameters. It is defined that if return water is treated by different electrodes, then $p H$ and $E h$ characteristics vary within the ranges from 3.2 up to 7.0 and from -500 up to $+1020 \mathrm{mV}$; in this context, Eh shifts towards the range of negative values making it possible to apply widely physical and chemical pulp characteristics. While changing physical and chemical properties of the return water in wide range, the obtained results allow developing such a water composition in terms of which disintegration process and water penetration into the rocks will increase by several time. In particular, if water system composition is turned to be acid, then disintegration process and water penetration into the ore samples will grow by $2-3$ times [6, 7]. Results of the laboratory studies are confirmed by industrial tests performed under conditions of factory \#3 [8]. It is identified that ore and rock breaking inside WSGM is more intense in terms of process water treatment in electrolysis units before its supply into WSGM; that indicates considerable effect of water properties upon ore softening.

\section{Conclusions}

Thus, ore disintegration in WSGM is effected considerably by water medium characteristics $p H$ and $E h$ which values vary within the ranges from 3.2 up to 7.0 and from -500 up to $+1020 \mathrm{mV}$. In this context, $E h$ shifts towards the range of negative values making it possible to vary significantly physical and chemical pulp characteristics and changing its composition so that disintegration process and water penetration into the ore will increase by several times.

\section{References}

1. Monastyrsky, V. F., Soloviev, S. V. (2004). Optimization of the modes of self-grinding mills during the enrichment of diamond-containing raw materials. Gornyi Zhurnal: Izvestiya VUZov, (6), 45-49

2. Firm Kluff starts to carry out the project of processing of diamonds in Copeton (1990). Mining Mag., (1), 62-68

3. Monastyrsky, V. F., Vorontsov, V. S. (2012). Models of diamond-bearing ore disintegration in the aquatic environment of wet self-grinding mills. Geo-Technical Mechanics, (104), 146-159

4. Goryachev, B. E. (2010). Tekhnologiya almazosoderzhashchikh rud. Moskva: MISIS

5. Langelier, W. F. (1946). Chemical Equilibria in Water Treatment. Journal of the American Water Works Association, (38), 169-181

6. Feodosev, V. I. (1999). Soprotivlenie materialov, 2. Moskva: MSTU

7. Chanturia, V.A., Vigdergauz, V.E. (2008). Innovative technologies for processing technogenic mineral raw materials. Gornyi Zhurnal, (6), 71-74

8. Dvoichenkova, G. P., Chernysheva, E. N., Savitsky, L. V., Vorontsov, V. S. (2009). Intensification of the processes of ore preparation and heavy-medium separation of diamondcontaining raw materials of the Nyurbinskaya tube. Gornyi Zhurnal, (6), 72-75 